\title{
Topology Reconfiguration for Optimization of Photovoltaic Array Output th
}

\author{
H. Braun ${ }^{\mathrm{a}, *}$, S. T. Buddha ${ }^{\mathrm{a}}$, V. Krishnan ${ }^{\mathrm{a}}$, C. Tepedelenlioglu ${ }^{\mathrm{a}}$, A. Spanias ${ }^{\mathrm{a}}$, M. Banavar ${ }^{\mathrm{b}}$, D. Srinivasan ${ }^{\mathrm{a}, \mathrm{c}}$ \\ ${ }^{a}$ SenSIP Center, School of ECEE, Arizona State University, Tempe, AZ 85287, USA \\ ${ }^{b}$ Department of Electrical and Computer Engineering, Clarkson University, Potsdam, NY 13699 \\ ${ }^{c}$ Poundra LLC, 2440 W. 12th Street, Suite 1, Tempe, AZ 85281
}

\begin{abstract}
As more utility scale photovoltaic (PV) power plants are installed, there is a need to improve monitoring and management of PV arrays. A procedure is presented here for optimizing the electrical configuration of a PV array under a variety of operating conditions. Computer simulations and analysis with synthetic and real data are presented in this paper. The performance of the optimization system is evaluated for a variety of partial shading conditions using a SPICE circuit simulator. In general, a $4-5 \%$ gain in power output is achievable by employing active switching in order to reconfigure the array's electrical configuration.
\end{abstract}

Keywords: Photovoltaic Systems, Fault Tolerant Systems, Condition Monitoring

\section{Introduction}

In spite of its many advantages, photovoltaic (PV) technology faces various barriers which prevent efficient utilization of this important technology. The major barrier is cost. In the United States in 2010, the average levelized cost of energy (LCOE) for PV electricity was $\$ 211 / \mathrm{MWh}$, while the LCOE of coal was only $\$ 95 / \mathrm{MWh}[1]$. PV has overcome the cost problem with conventional energy only for special cases such as very remote locations where fuel shipping costs are extremely high. The other barrier is its dependence on weather conditions, resulting in stability and reliability problems for the electrical grid. These issues suggest that any technology that would lower cost or improve reliability will increase the deployment and improve efficiency of utility scale PV power generation.

In this paper a method of optimizing the electrical configuration of a dynamically switchable PV array is presented. This system is developed as one part of a comprehensive PV monitoring system incorporating performance analysis, fault detection, and mismatch mitigation $[2,3,4,5,6]$. The optimization strategy results in higher power output under array mismatch conditions such as partial shading. It is shown that the optimal electrical configuration under partial shading varies depending on

\footnotetext{
This work is supported in part by the NSF GOALI award 1308052, the NSF Net Centric I/UCRC - ASU SenSIP site award 1035086, Poundra Inc, ViaSOL Inc, ACT Corporation, and Paceco Corp.

*Principal Corresponding Author

Email addresses: hcbraun@asu.edu (H. Braun), sbuddha@asu.edu (S. T. Buddha), vkrish20@asu.edu ( V. Krishnan), cihan@asu.edu (C. Tepedelenlioglu), spanias@asu.edu (A. Spanias), mbanavar@clarkson.edu (M. Banavar), dsriniva@asu.edu (D. Srinivasan)
}

which modules are shaded, motivating the development of an optimization algorithm. Power increases of $4-6 \%$ are observed by switching from a base configuration to the optimal configuration.

The paper is organized as follows: in Section 1 the operation of PV arrays is described, including under partial shading conditions, and in Section 3 several approaches to the problem of maximizing output under shading conditions are described. The reconfiguration method is described in Section 4. Section 5 describes the simulations used to test the method and their results. Finally, the significance of the optimization method and its usefulness is discussed in Section 6.

\section{Problem statement}

This section provides an outline of the topology reconfiguration system and discusses the physics behind the operation of a PV array. The various topologies that are employed in practice and their electrical behavior is presented. The models that can be used to predict the electrical characteristics of a PV module are explained.

\subsection{Electrical Behavior of a PV Module}

A typical PV cell consists of a P-N junction in silicon or another semiconductor, although more exotic configurations exist [7]. Although a single cell is capable of generating significant current, it operates at an insufficient voltage for typical applications. To obtain a higher voltage, cells are connected in series and encapsulated into a PV module/panel. These modules show similar electrical behavior to individual cells, with the exception of small effects due to mismatch between cells.

Fig. 1 shows the commonly used single-diode model of a PV module. At the heart of the device is a current source 


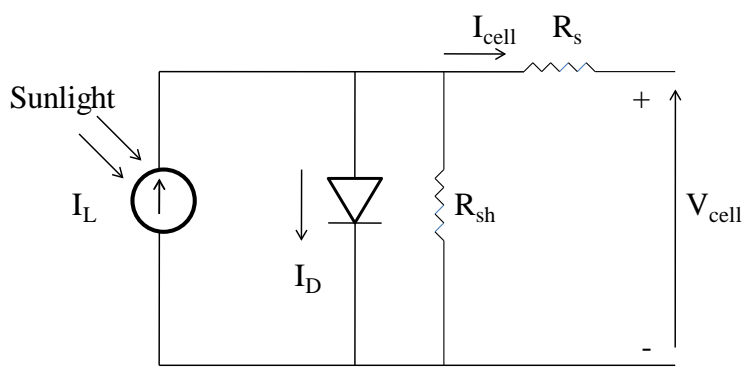

Figure 1: Equivalent circuit of a solar cell including parasitic resistances.

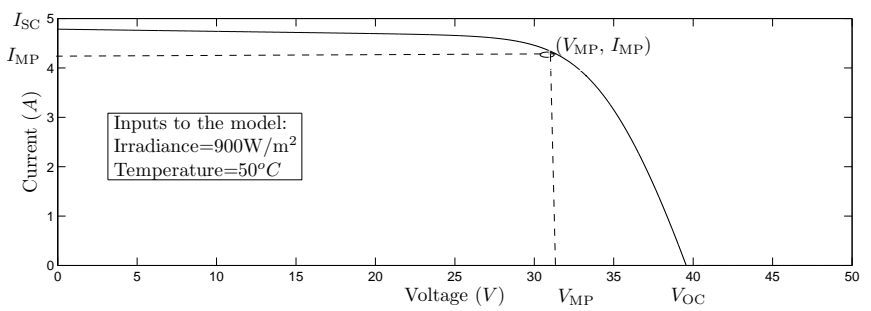

Figure 2: I-V characteristic of a photovoltaic module.

$I_{L}$ connected to a diode. $I_{L}$ models the current generated by incoming light while the diode's current models the action of the P-N junction and limits the power output of the cell. Some of the power generated by the solar cell is dissipated through parasitic resistances. These resistive effects are electrically equivalent to a series resistance $R_{\mathrm{S}}$ and a parallel ("shunt") resistance $R_{\text {sh }}[8]$.

Like a diode, a PV cell or module is defined by its current-voltage (I-V) characteristic. An example of the I$\mathrm{V}$ curve of a photovoltaic module is shown in Fig. 2. In addition to the short-circuit current $I_{\mathrm{SC}}$ and open-circuit voltage $V_{\mathrm{OC}}$, another important point on the $\mathrm{I}-\mathrm{V}$ curve is the maximum power point (MPP). This is the point $\left(V_{\mathrm{MP}}, I_{\mathrm{MP}}\right)$ at which the power of the array is maximized. PV inverters adjust the electrical load they present to the $\mathrm{PV}$ array in order to maintain operation at the maximum power point.

\subsection{The UW-Madison PV Model}

The UW-Madison PV performance model, developed at the Wisconsin Solar Energy laboratory [8], is adopted for simulations in this paper. It was chosen for several reasons, the most important being its accurate modeling of silicon PV modules. It also gives an explicit electrical circuit representation of a module using only data provided by the module manufacturer at standard test conditions. This allows the UW-Madison model to be used on any conventional silicon PV module. In contrast, the widely used and highly accurate Sandia model [9] requires extensive measurements of each new design and does not give an explicit circuit representation. The UW-Madison model depends on the light generated current $I_{\mathrm{L}}$, the diode reverse saturation current $I_{0}$, the series resistance $R_{\mathrm{s}}$, the shunt (parallel) resistance $R_{\mathrm{sh}}$, and the modified ideality factor $a$ [10]. The modified ideality factor is given by

$$
a=\frac{N_{\mathrm{s}} n k T_{\text {cell }}}{q}
$$

where $q$ is the electron charge, $k$ is the Boltzmann constant, and $T$ is the absolute temperature of the cell. $n$ is the diode ideality factor and typically takes values between 1 and $3 . N_{\mathrm{s}}$ represents the number of cells in series.

From Fig. 1, the current generated by the photovoltaic module $\left(I_{\mathrm{mod}}\right)$ is given as [8],

$$
\begin{aligned}
I_{\text {mod }} & =I_{\mathrm{L}}-I_{0}\left[\exp \left(\frac{V_{\text {mod }}+I_{\text {mod }} R_{\mathrm{s}}}{a}\right)-1\right] \\
& -\frac{V_{\text {mod }}+I_{\text {mod }} R_{\mathrm{s}}}{R_{\mathrm{sh}}}
\end{aligned}
$$

where $V_{\text {mod }}$ is the terminal voltage of the module. The model is semi-empirical in that it calculates parameters by fitting manufacturers' measured module data to a physicsbased model of a PV cell. An easy to use application to determine the reference parameters was developed by the University of Wisconsin [11]. A sample I-V curve of the UW-Madison model implemented in MATLAB is shown in Fig. 2.

\subsection{Effect of Temperature and Irradiance}

The power output of solar cells is significantly affected by variations in the temperature and irradiance. The most significant effect of temperature is on the cell terminal voltage $V_{\text {cell }}$. This effect is driven by the dependence of diode current $I_{\mathrm{D}}$ on temperature $T_{\text {cell }}$, as shown in (2). It decreases with increase in temperature, i.e it has a negative temperature coefficient.

The light generated current $I_{\mathrm{L}}$, and therefore the short circuit current $I_{S C}$, are typically modeled as being directly proportional to the irradiance. The voltage variation is much smaller due to its logarithmic dependence on the irradiance and is usually neglected in practical applications $[12]$.

\subsection{Alternate Topologies}

Photovoltaic cells are electrically connected to form a photovoltaic module. Photovoltaic modules are typically connected in series-parallel combinations to form a photovoltaic array as shown in Fig. 3.

Apart from the series-parallel combination, the modules can also be connected in a cross-tied manner in which additional connections are introduced between the modules. Two types of cross-tied topologies are discussed in the literature: the total cross-tied (TCT) topology and the bridge link (BL) topology. In the total cross-tied topology shown in Fig. 4, each of the PV modules is connected in series and parallel with the others [13]. The bridge link topology shown in Fig. 5 consists of half as many interconnections as the TCT topology [13]. When there are 


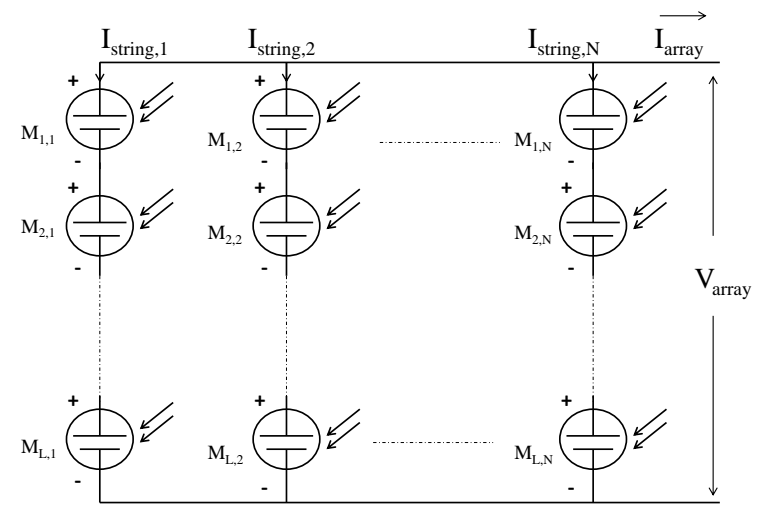

Figure 3: Photovoltaic modules connected in series-parallel (SP) configuration.

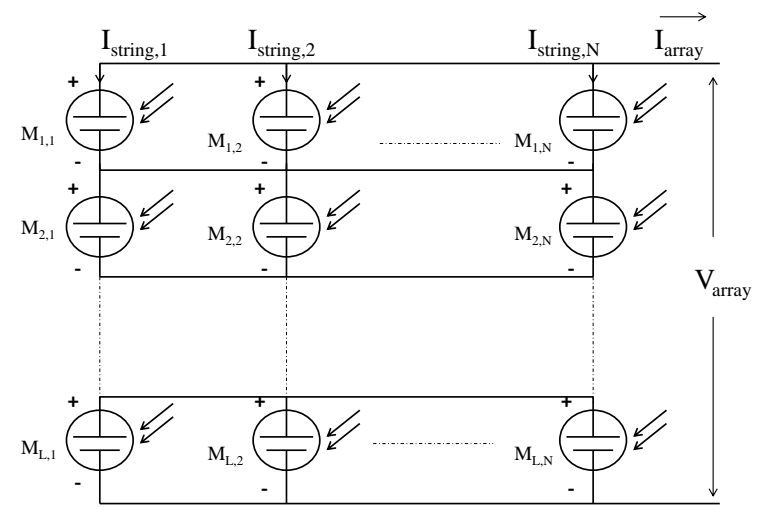

Figure 4: Photovoltaic modules connected in total cross-tied (TCT) configuration.

no wiring losses or module mismatches, all the modules behave identically and the generated array power is the same for all three topologies. When there are electrical mismatches, it will be shown that one of the topologies outperforms the others.

\subsection{Constraints on Feasible Configurations}

The design methodologies for PV arrays are specified in [14]. The number of modules in a series string is limited by the operating range of the inverter. The maximum voltage that can be generated by the array should not exceed the maximum input voltage to the inverter. Since the module voltage increases at lower temperature, the open circuit voltage of the inverter must not surpass the maximum operating voltage of the inverter on the coldest day of the year. The maximum number of modules $N_{\max }$, that can be connected in a string is given by

$$
N_{\max }=\frac{V_{1}}{V_{2}}
$$

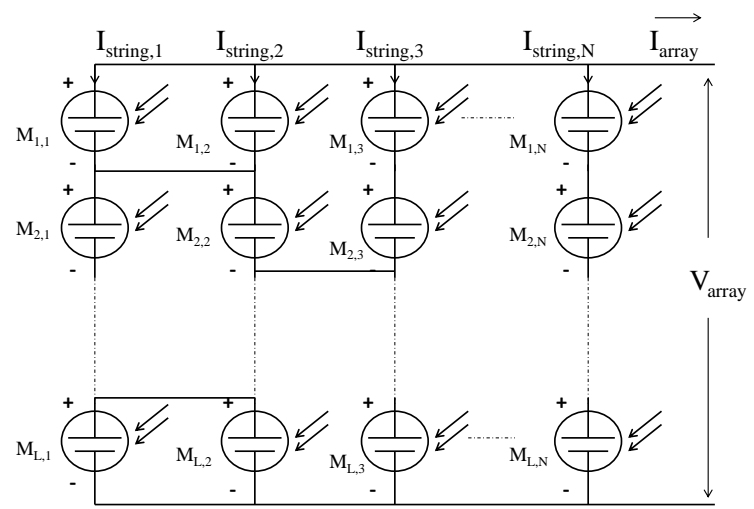

Figure 5: Photovoltaic modules connected in bridge link (BL) configuration.

where $V_{1}$ is the maximum allowable input voltage of the inverter and $V_{2}$ is the open circuit voltage of a module at the lowest winter temperature of the year.

The number of modules $N_{\min }$ that can be connected in a string is determined by the minimum input voltage requirement of the inverter and the maximum temperature at which the modules need to operate. It is given as

$$
N_{\min }=\frac{V_{3}}{V_{4}}
$$

where $V_{3}$ is the minimum input voltage of the inverter and $V_{4}$ is the maximum power voltage $V_{\mathrm{MP}}$ of a module at the highest module temperature during the year. Other factors such as the efficiency of the inverter at different voltages can be considered to determine the exact number of modules in a string.

The limitation on the number of strings that can be connected in parallel is determined by the maximum input current to the inverter and the current carrying capacity of the wires used. The maximum number of strings in parallel $N_{\mathrm{P}}$ is given by

$$
N_{\mathrm{P}}=\frac{I_{1}}{I_{2}},
$$

where, $I_{1}$ is the maximum current that can be input to the inverter, $I_{2}$ is the short circuit current at maximum irradiance for the given string.

In the US, the National Electric Code [15] governs the tolerance levels for the current carrying wires used in the construction of PV arrays. The code requires the wires used to be rated for at least $156.25 \%$ of the maximum short circuit current they might expected to carry. This further constrains the maximum number of strings that can be kept in parallel in addition to the restriction imposed by (5). 


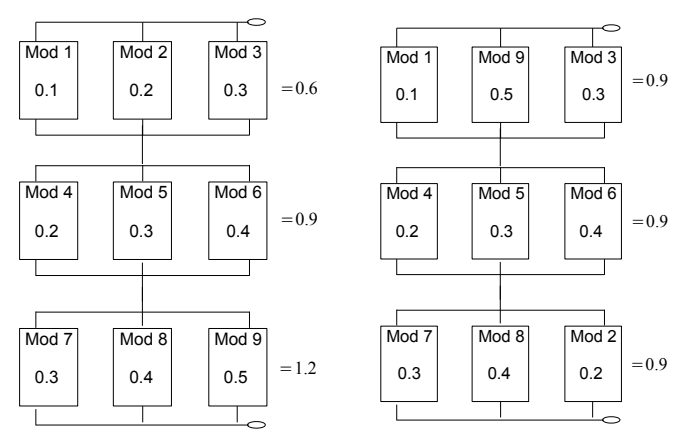

Figure 6: Demonstration of the Irradiance Equalization method.

\section{Previous Work}

The major problems that cause the PV array to generate power less than the rated power are shading and improper selection of topology. To overcome the shading problem, several methods have been proposed. The techniques and their associated drawbacks are discussed below.

\subsection{Irradiance Equalization}

The irradiance equalization method $[16,17,18]$ is applied to PV arrays that are connected in total cross-tied (TCT) configuration (discussed in Section 3.3). Depending on the irradiance received by the modules, they are relocated such that each row in the series string has similar irradiance.

A shaded module, when present in a string, limits the current in the string to the lower current of the shaded module. By connecting PV modules with similar operating characteristics in a string, power output is maximized. The concept of irradiance equalization is shown in Fig. 6 . The PV array consists of 9 modules connected in the TCT configuration. Each row in the figure consists of 3 modules connected in parallel and can together be considered as a single block module. The current generated by the block module is the sum of the currents of the individual modules. Three such block modules are connected in series. The normalized effective irradiance (relative to standard irradiance of $1000 \mathrm{~W} / \mathrm{m}^{2}$ ) of each module is estimated from the measured voltage and current using the ideal PV cell equation.

The block effective irradiance is the sum of the individual module effective irradiances. Fig. 6 (a) shows block modules with unmatched effective irradiance factors. The switching matrix is then used to rearrange the electrical location of the modules 2 and 9 and the irradiance matched configuration is shown in Fig. 6 (b). The output for the configuration in Fig. 6 (b) is found to be higher. This is due to the fact that the current mismatch between the blocks is reduced before connecting them in series. This method is applied on an array connected in a a fixed total cross-tied topology. Though there is reconfiguration within the topology, it might not be the optimal topology.

\subsection{Adaptive Banking}

The adaptive banking method $[19,20]$ reconfigures the PV array to provide maximum power output under different shading conditions. Here the PV system has two parts: the fixed part and the adaptive part. The fixed part constitutes the PV array that is connected in total cross-tied (TCT) configuration. The adaptive part consists of a bank of individual PV modules. When the power output of the PV array (fixed part) goes down, the modules from the adaptive bank are connected in parallel to modules in the fixed part. This is accomplished by employing a switching matrix constructed using relays or electrical switches. The most illuminated solar module from the adaptive bank is connected in parallel to the row of the fixed part that has the least power output (the most shaded row). In this manner all the modules from the adaptive bank are connected.

Consider an array of 16 modules connected in the total cross-tied (TCT) configuration as shown in Fig. 7 (a). Under normal operating conditions there is no need for any changes to the configuration. However, three of the modules in the array are severely shaded. The maximum current that can be generated by this array is now limited by the first row, which has only two healthy modules. This detrimental effect can be minimized to a certain extent by using the adaptive banking method. Here, the first three columns can be made the fixed part and the last column, the adaptive part. Each module in the adaptive part can be connected to any of the rows of the fixed part. With this arrangement, the severely mismatched condition of rows in Fig. 7 (a) can be rectified as shown in Fig 7 (b). In this case, two modules from the adaptive part of the array are added in parallel to the first row which has 2 modules shaded and one module is added to the second row which has one shaded module. Now, each row in the array has at least three healthy modules, therefore the current is not restricted to two modules as in the previous case. If the mismatch is not severe, the most illuminated solar module from the adaptive bank is connected in parallel to the row of the fixed part that has the least power output (the most shaded row).

This method also employs the fixed total cross-tied topology and switching matrix to increase the efficiency of the array. Compared to the irradiance equalization method, it requires a switching matrix of smaller size as it relocates only the modules in adaptive bank rather than all the modules in the array. However, it still uses a nonoptimal fixed array topology and needs an additional set of modules allocated as the adaptive bank. The additional modules increase the cost of the system.

\subsection{Alternate Topologies}

Alternate module interconnection schemes are suggested in [13] to overcome mismatch losses, especially under shading conditions. In [21], the TCT topology is shown to be the optimal topology for all possible shading patterns in 


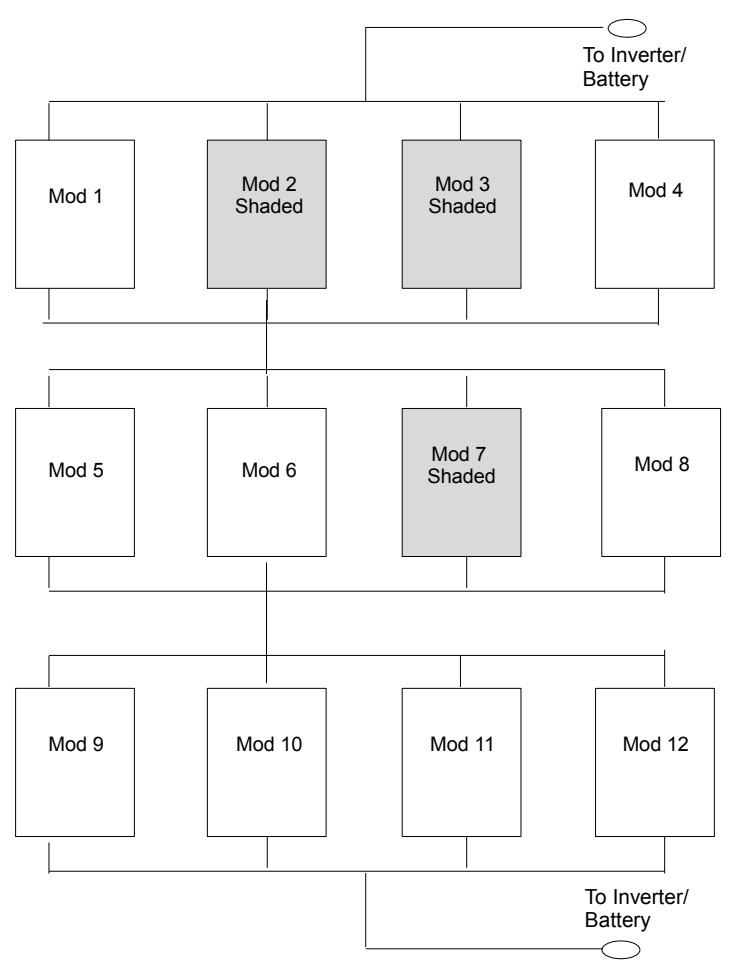

(a) Array before irradiance equalization.

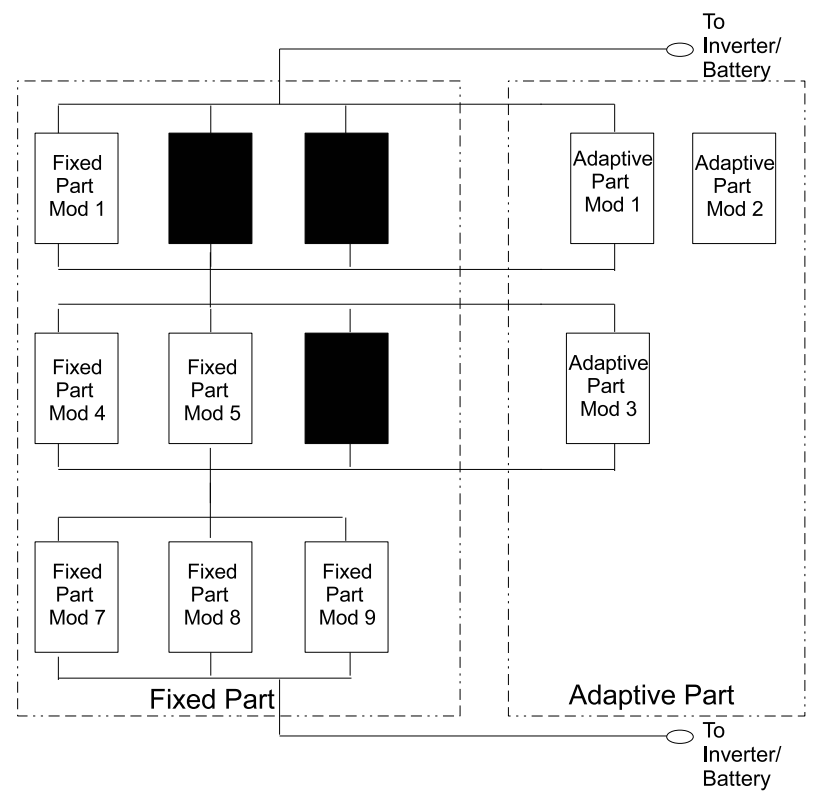

(b) Array after irradiance equalization.

Figure 7: Demonstration of the Adaptive banking method. an array consisting of four PV modules. It was experimentally shown in [22] that in an array with two shaded modules, changing the traditional series-parallel configuration to bridge link (BL) and total cross-tied (TCT) configurations resulted in a $4 \%$ increase in the array power under shading conditions. Cross-tied topologies such as TCT and BL are shown to be more tolerant to mismatch losses caused due to aging and manufacturing process tolerances in $[23,24]$. It has been shown experimentally that the cross tied topologies perform well for a simulated case of two shaded modules [22]. However, it is not guaranteed that the cross tied topologies alone would outperform all the existing topologies for any number of shaded modules (or any shading pattern) in the array.

This research proposes dynamic reconfiguration of the array topology under shading to extract the maximum yield from the array. The topology reconfiguration method is used to find the optimal topology for given weather conditions and faulty module information. The efficiencies of the existing topologies along with a new bypassed and reconfigured topology are analyzed for various shading patterns.

\section{Topology Reconfiguration Algorithm}

In this section, the topology reconfiguration method used to find the optimal array topology under shading conditions is described. An ideal PV array connected in any topology performs identically. When faults occur in the array, however, the performance of each of the topologies differs and the default array topology may not produce the maximum yield. In this section, a topology reconfiguration method is proposed to predict the optimal topology for a photovoltaic array consisting of shaded modules. This method assumes that the PV system is provided with an array reconfiguration facility. The topology reconfiguration method can be implemented in monitoring systems to predict the best suitable array configuration for a definite time period using the previous array's measurements.

The topology reconfiguration method is described in the block diagram shown in Fig. 8. The operation of the system is explained below.

\subsection{Fault Detection Algorithm}

A monitoring system provides the plane of array (POA) irradiance and the module level measurements: the maximum power point voltage $\left(V_{\mathrm{MP}}\right)$, maximum power point current $\left(I_{\mathrm{MP}}\right)$, module temperature and the aging information, which can be used to detect faults using the fault detection algorithms. In general, the faulty modules form a cluster in the $\mathrm{I}-\mathrm{V}$ space, while the rest of the string in which the fault occurs forms another cluster, and the remaining unaffected modules in the string form a third cluster. One such algorithm to detect the shaded modules is explained in $[2,3]$. 


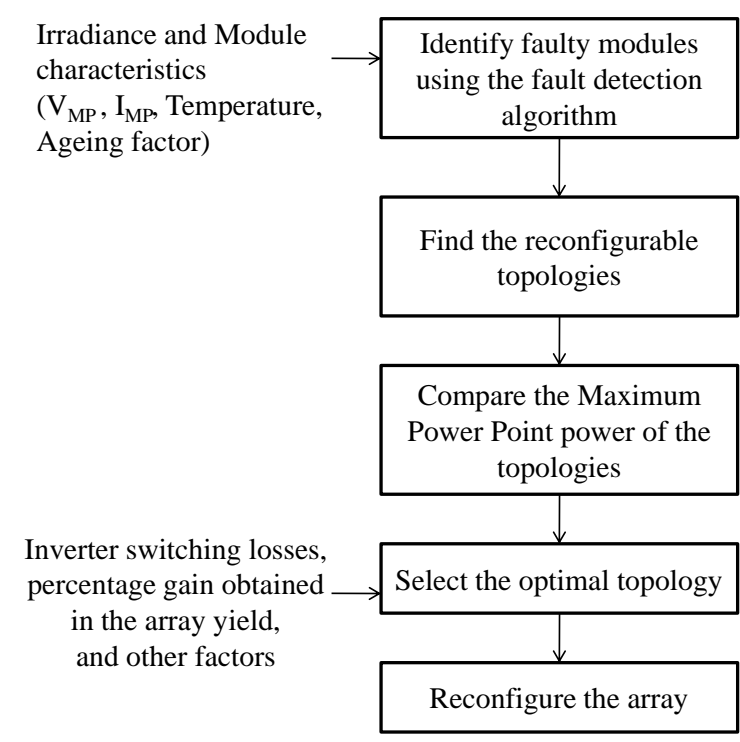

Figure 8: Block diagram explaining the topology reconfiguration method.

\subsection{Feasible Configurations}

The information from the fault detection algorithms is also used to find the feasible topology/topologies. For example in the case of shading, the faulty modules can be bypassed based on the intensity of shading. A new configuration is obtained by bypassing the shaded modules. This topology can also be reconfigured by evenly distributing the modules into a row-column array resulting in another topology. Therefore SP, BL, TCT, and the bypassed and reconfigured (BR) topologies constitute the feasible configurations.

\subsection{Performance of the Topologies}

Once the feasible configurations are determined, their performance can be predicted using any of several PV performance models [8, 9]. For example, the direct current (DC) power generated by a topology can be determined for a given irradiance $\left(\mathrm{W} / \mathrm{m}^{2}\right)$ and module temperature $\left({ }^{\circ} \mathrm{C}\right)$ using the UW-Madison model in a circuit simulator such as SPICE. The maximum power point (MPPT) power can be used as a performance metric to compare the topologies. The design of the topologies and procedure to find the MPPT power is explained in Section 5.1. The MPPT power is expressed as a percentage power obtained with respect to the array power under ideal (no fault) conditions.

\subsection{Additional Considerations for Optimization}

Several factors beyond simulated MPPT power affect the decision to switch array topology. Most notably, switching losses reduce the effectiveness of the algorithm. This occurs because the IEEE 1547 standard [25] mandates a five-minute shutdown following any out-of-bounds condition. Rapid restart is limited by standards compliance rather than technical challenges; some non-grid-interacting inverters [26, 27] feature an automatic restart function following power interruption. Note that this switching delay is caused entirely by the inverter; the optimization algorithm itself only simulates a small number of feasible configurations and does not require significant time or computational resources to run. The current version is not optimized for runtime in any way and requires less than 3 seconds to run on a desktop computer.

The simulation results that follow in Section 5 consider only MPPT power on the DC side of the inverter to determine the optimal topology. Inverter down-time, resistive wiring losses, and variable $\mathrm{AC} / \mathrm{DC}$ conversion efficiency are not considered. Of these effects, only inverter down time is likely to significantly impact results. The authors are currently studying the prediction of future irradiance at the module level, with the goal of providing an optimal decision on when to perform reconfiguration.

\section{Simulation Results}

This section explains the simulation set-up used to carry out the simulations. The simulation model is validated with experimental data collected from a prototype monitoring system installed at the Solar Testing and Research (STAR) Center in Tempe, Arizona. The behavior of the topologies in the presence of various shading scenarios is presented. The topology reconfiguration method is used to find the best topology for each of the shading patterns. The effect of irradiance and shading on the array performance is studied.

\subsection{Simulation Set-up and experimental validation}

This section describes the experimental set-up of the PV array followed by a comparison of the simulated results with the experimental data. A PV array of 52 modules arranged in a 13 series, 4 parallel $(13 \times 4)$ configuration was used for the simulations. Parameters for the Sharp NT-175U1 module and Satcon PowerGate 50kW inverter were used. The array was simulated with the UW-Madison model discussed in Section 2.1 in a SPICE circuit simulator.

The weather information and module temperatures correspond to Phoenix area for the day May 3rd, 2011 for all the simulations that follow in this section. The array performance is found for the day and an average measure is taken to compare the performance of topologies.

A grid connected photovoltaic array was established in Tempe, Arizona to study the benefits of topology reconfiguration. The array was constructed as described in Section 5.1 using 52 Sharp NT-175U1 modules. The array was equipped with a basic switching system which allowed the implementation of arbitrary TCT topologies. Each module was equipped with sensors which reported its voltage, current, and module back surface temperature. This 


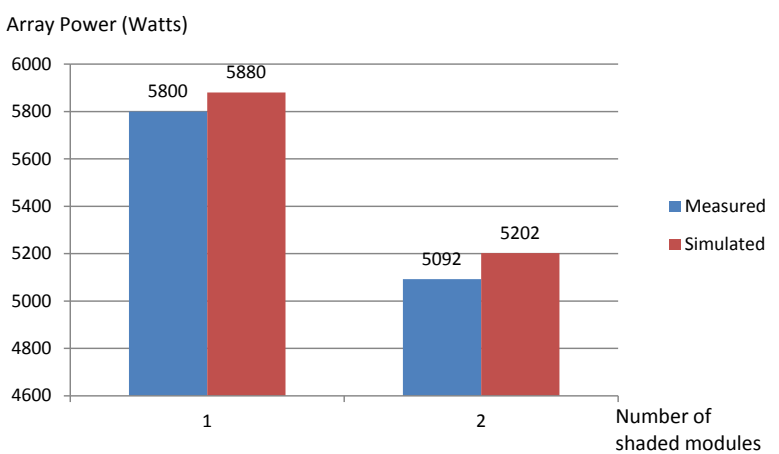

Figure 9: Comparison of the measured and simulated data.

information was recorded at a central server along with measured irradiance values.

Experimental data for the series-parallel (SP) topology in $13 \times 4$ configuration are available from the prototype monitoring system. Array data for two shading tests was used to validate the simulation results. In the first test, a single module was shaded in a string of the array. In the second test, two modules were shaded in a string. A mesh that corresponds to a shade factor of $58.6 \%$ was used to cover the modules and introduce shading. The simulations were performed in a SPICE simulator using the provided irradiance and module temperature measurements. Fig. 9 compares the simulated and measured (experimental) results.

From the comparison between the simulated and measured data in Fig. 9, it is seen that the simulated results match closely with the experimental data. The small deviation of approximately $2 \%$ may be due to the wiring losses or module aging. In the simulations, wires are assumed to be ideal and modules are expected to perform without any aging effect.

\subsection{Topology Reconfiguration under Shading}

In this section, the topology reconfiguration method is applied to find the optimal topology. The behavior of the topologies for various shading patterns is studied. Here only the MPPT power is used to determine the optimal topology. The array performance is studied on the DC side of the inverter, so inverter switching losses need not be considered. The array is assumed to have negligible resistive wiring losses.

\subsubsection{Shading Pattern-1}

In the first shading pattern, modules are shaded in a single string of the photovoltaic array. This shading pattern is analogous to the shadow caused by trackers, overhead power lines, etc. The shading pattern for a SP topology with seven shaded modules is shown in Fig. 10. A shade factor of $58.6 \%$, corresponding to the shade offered by a semitransparent mesh used in testing, is considered. Shade factor is defined here as the fraction of total unshaded irradiance still illuminating the shaded module.

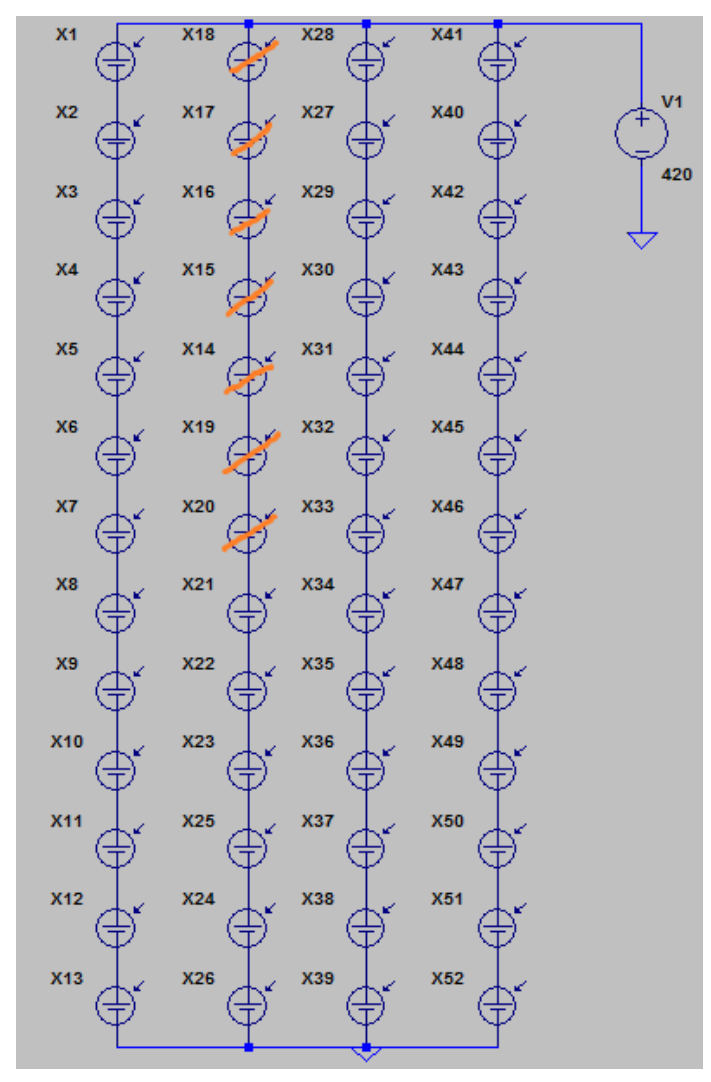

Figure 10: Illustration of shading pattern-1. 


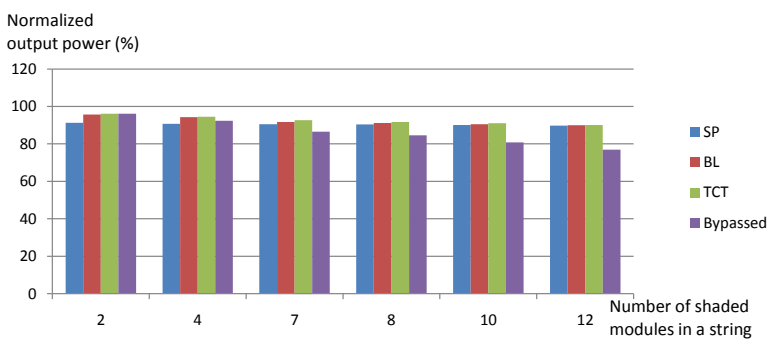

Figure 11: Performance of topologies for different number of shaded modules for the shading pattern-1.

\begin{tabular}{|c||c||c||c||c|}
\hline \multicolumn{1}{|c||}{ N } & \multicolumn{3}{c|}{ Percentage output power generated by the array } \\
\hline & SP & BL & TCT & BR \\
\hline 2 & 91.3 & 95.7 & 96.12 & $96.14(10 \times 5 \mathrm{SP})$ \\
4 & 90.7 & 94.2 & 94.5 & $92.3(12 \times 4 \mathrm{SP})$ \\
7 & 90.5 & 91.6 & 92.6 & $86.5(15 \times 3 \mathrm{SP})$ \\
8 & 90.3 & 91.1 & 91.6 & $84.6(11 \times 4 \mathrm{SP})$ \\
10 & 90.1 & 90.4 & 91.0 & $80.7(14 \times 3 \mathrm{SP})$ \\
12 & 89.7 & 89.9 & 90.0 & $76.9(10 \times 4 \mathrm{SP})$ \\
\hline
\end{tabular}

Table 1: Performance of the topologies for the shading pattern-1.

Two modules are shaded in the second string of all the topologies. The bypassed and reconfigured topology (BR) is obtained by bypassing the two shaded modules (i.e. leaving one terminal floating so that the module operates at open circuit conditions) and reconfiguring the array to minimize mismatch between remaining modules. The number of shaded modules $(\mathrm{N})$ in the second string is increased to 4,7 and 10 to observe the trend in the performance of the topologies. The topology reconfiguration method is used to find the optimal topology in each of the cases. Fig. 11 and Table 1 show the percentage output power of the topologies, expressed in reference to the array output under ideal (no shade) conditions.

From the results, it is observed that when there are more than 2 shaded modules, the TCT configuration performs best. For the case of two shaded modules, switching the topology from SP to TCT and BR topologies results in a power increase of $4.82 \%$ and $4.84 \%$, respectively. While this indicates that the BR configuration is optimal, the difference between the BR and TCT configurations is negligible. Because of this, the TCT topology is considered the optimal topology for this shading pattern.

\subsubsection{Shading Pattern-2}

In the second shading pattern, modules are shaded across the strings, a pattern which is equivalent to the shadow of chimneys, trees etc. The shading pattern for a SP topology with seven shaded modules is shown in Fig. 12. A shade factor of $58.6 \%$ is once again used.

For example, say seven modules are shaded with two modules each in the first three strings and one in the fourth string. Using the topology reconfiguration method, it is observed that the bypassed and reconfigured topo-

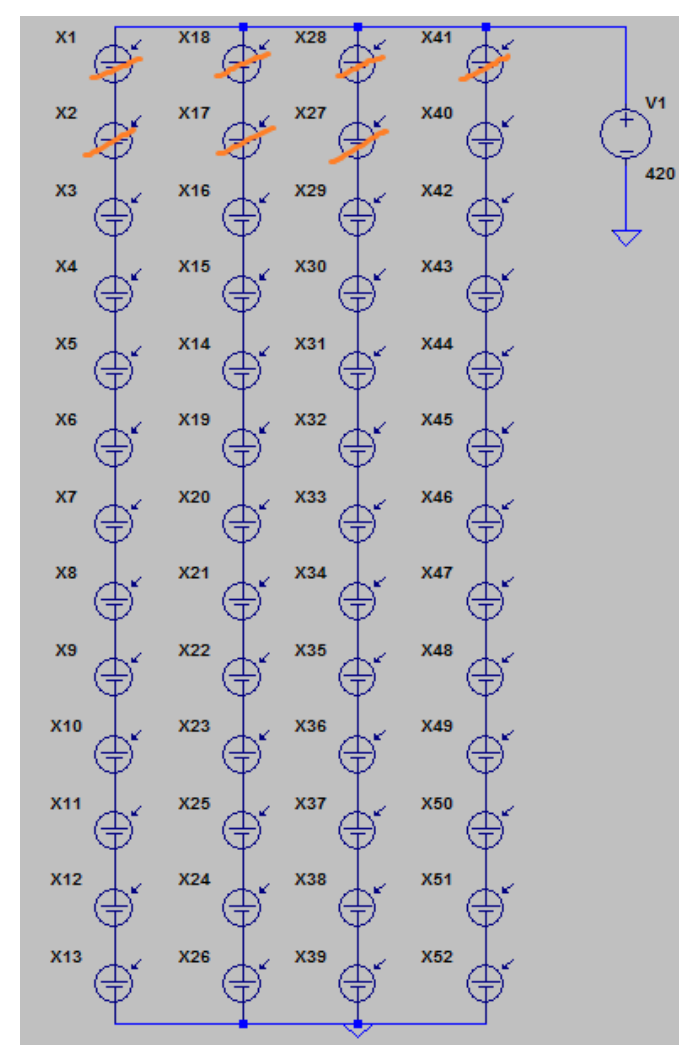

Figure 12: Illustration of shading pattern-2.

\begin{tabular}{|c||c||c||c||c|}
\hline \multicolumn{1}{|c||}{ N } & \multicolumn{4}{c|}{ Percentage output power generated by the array } \\
\hline & SP & BL & TCT & BR \\
\hline 7 & 85.5 & 84.5 & 84.3 & $86.5(15 \times 3 \mathrm{SP})$ \\
10 & 79.0 & 77.1 & 76.6 & $80.7(14 \times 3 \mathrm{SP})$ \\
\hline
\end{tabular}

Table 2: Performance of the topologies for the shading pattern-2.

logy performs better than the rest of the topologies. It is followed by series-parallel topology, which performs better than both the cross tied topologies. A similar result is obtained when ten modules are shaded across the strings, e.g. when $M(1,1), M(2,1), M(3,1), M(1,2) M(2,2), M(3,2)$, $M(1,3), M(2,3), M(1,4)$, and $M(2,4)$ are shaded. The results are shown in Fig. 13 and Table 2. It is observed for this case that switching the topology from TCT to $\mathrm{BR}$ results in a $4.1 \%$ increase in the array output power. Therefore the bypassed and reconfigured (BR) topology is the optimal topology and reconfiguration of TCT to BR topology would result in a $\%$ gain of 4.1 in the array power relative to the TCT configuration.

\subsubsection{Shading Pattern-3}

In this shading pattern, modules are shaded in two strings of the array. The shading pattern for a SP topology with ten shaded modules is shown in Fig. 14. A shade factor of $40.0 \%$ is considered. 


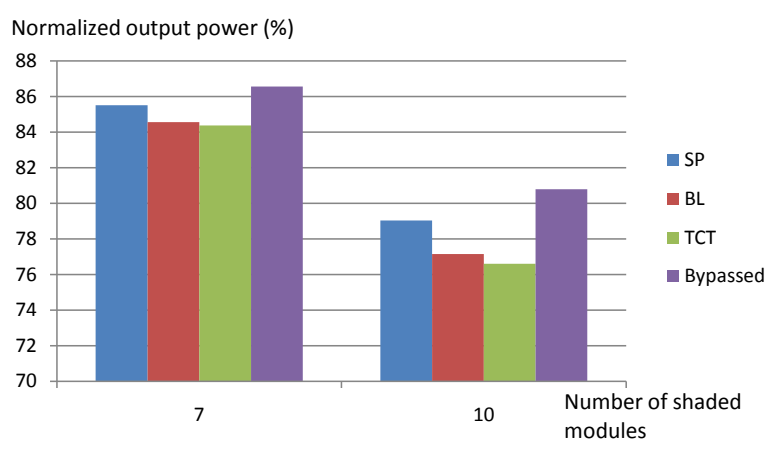

Figure 13: Performance of the topologies for the shading pattern-2.

\begin{tabular}{|c||c||c||c||c|}
\hline \multicolumn{1}{|c||}{ N } & \multicolumn{3}{c|}{ Percentage output power generated by the array } \\
\hline & SP & BL & TCT & BR \\
\hline 10 & 82.4 & 80.5 & 80.8 & $80.8(14 \times 3 \mathrm{SP})$ \\
12 & 71.5 & 74.6 & 77.3 & $76.9(10 \times 4$ SP $)$ \\
\hline
\end{tabular}

Table 3: Performance of the topologies for the shading pattern-3.

For example, say ten modules are shaded with nine modules and one module in the first and second strings respectively. The shaded modules are $M(1,1)$ to $M(9,1)$ and $M(1,2)$ in the first and second strings respectively. Similarly twelve modules are shaded with nine and three modules in the first and second strings respectively. The modules $M(1,1)$ to $M(9,1)$ and $M(1,2)$ to $M(3,2)$ are shaded. The results are shown in Fig. 15 and Table 3.

It is observed that when twelve modules are shaded, switching the topology from SP to TCT and BR topologies results in a power increase of $5.79 \%$ and $5.44 \%$, respectively, in the array output power. This shows that the total cross-tied topology (TCT) is the optimal topology in this case.

\subsubsection{Analysis}

From the results, it is clear that no one array topology is superior and the actual pattern of shading determines the optimal topology. For the first shading pattern, switching from series-parallel to bypassed and reconfigured topology results in a $4.84 \%$ increase in the array power. However, for shading pattern-2, switching from total cross-tied to bypassed and reconfigured topology results in an increase of $4.1 \%$ and for shading pattern-3, a $5.79 \%$ gain is seen by switching from series-parallel to total cross-tied topology. Therefore from the simulation results, reconfiguration of topologies under shading resulted in an increase in the array power by 4 to $6 \%$. This offers evidence that a topology reconfiguration method and facility to reconfigure the photovoltaic array would improve the yield from the photovoltaic array.

\subsection{Effect of Irradiance on the Array Performance}

The effect of irradiance on the performance of the topologies is studied by varying number of shaded modules. The

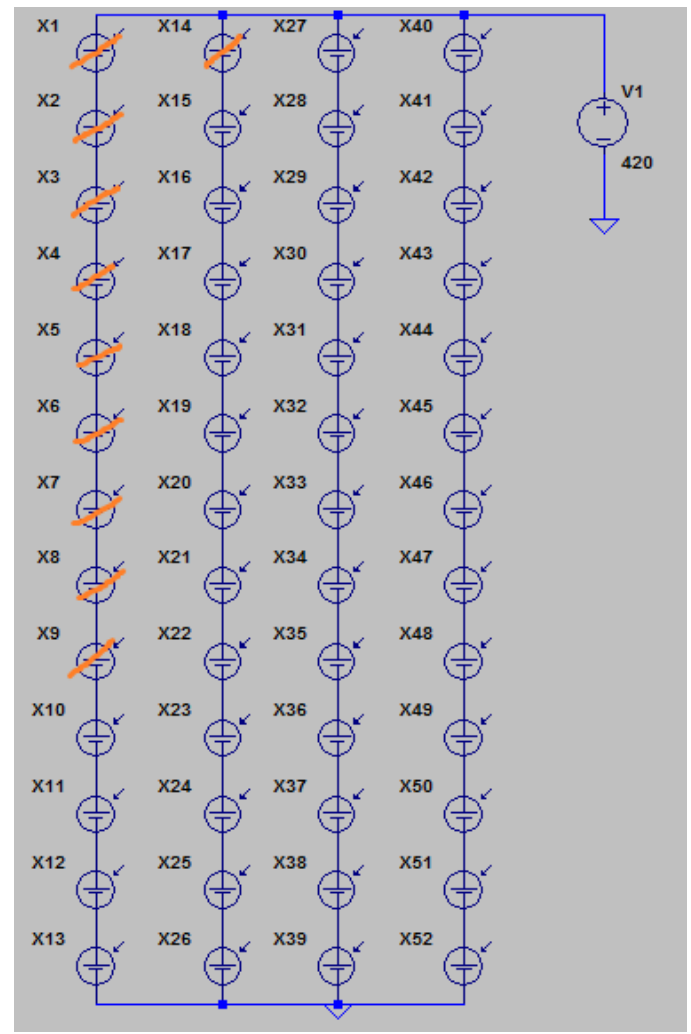

Figure 14: Illustration of shading pattern-3.

shading pattern shown in Fig. 10 is considered. The number of shaded modules is progressively increased from 2 to 12 . The irradiance values fall in the range 100 to 1000 $\mathrm{W} / \mathrm{m}^{2}$. The array behavior of the series-parallel(SP), total cross-tied (TCT), and bridge link (BL) topologies is studied and the results are shown in Figs. 16, 17, and 18. For all the topologies, it is observed that the percentage loss in array power for a given number of shaded modules does not vary significantly with respect to irradiance. For a given number of shaded modules, the maximum percentage deviation in any of the topologies is $1.5 \%$. Similar results are obtained irrespective of the shade factor and the type of shading pattern.

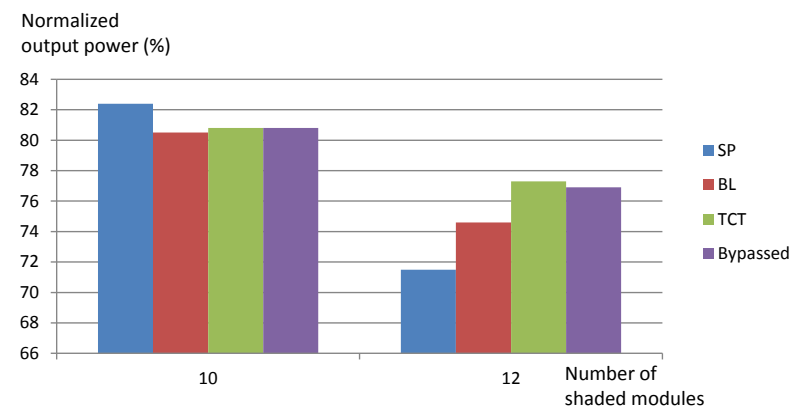

Figure 15: Performance of the topologies for the shading pattern-3. 
Normalized

output power (\%)

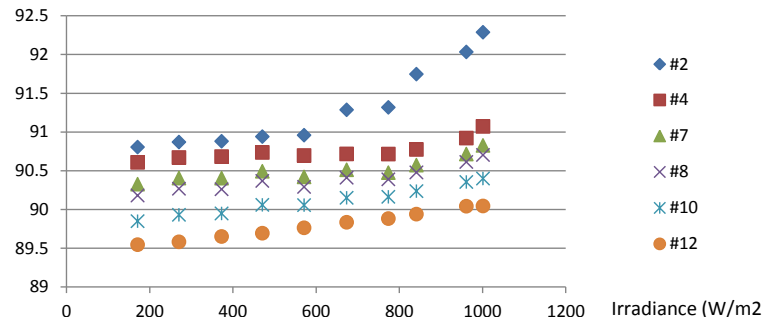

Figure 16: Effect of Irradiance on the performance of SP topology.

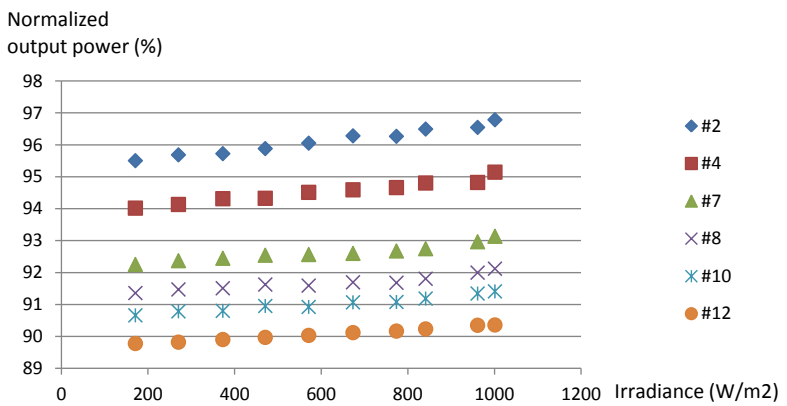

Figure 17: Effect of Irradiance on the performance of TCT topology.

\subsection{Effect of Intensity of Shading on the Array Perfor- mance}

The effect of intensity of shading is studied by progressively increasing the shade factor from 10 to $100 \%$ to include the possible range of shading. The performance of series-parallel, total cross-tied, and bridge link topologies is found for the various feasible shading patterns containing five shaded modules. The optimal topology for each of the shading patterns is analyzed. The simulation results, analysis, and the generalized results are explained in this section.

\subsection{Simulation Results}

The shading pattern (a), shown in Fig. 19, consists of shaded modules present in a single string. The performance of the topologies for the various shade factors is found and the percentage output power generated by the array with reference to the array power at ideal conditions is given in Table 4 . It is observed from the results

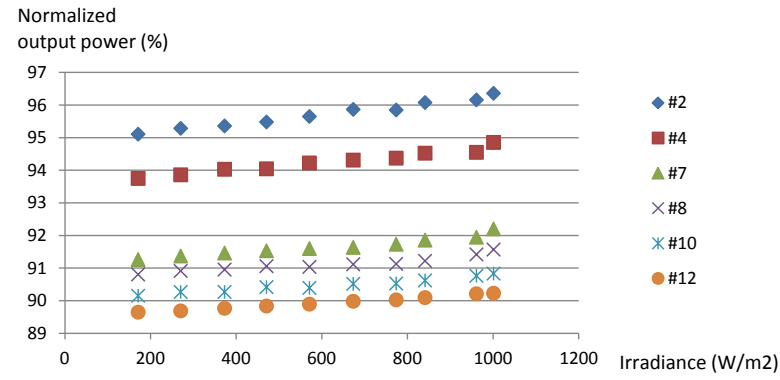

Figure 18: Effect of Irradiance on the performance of BL topology.

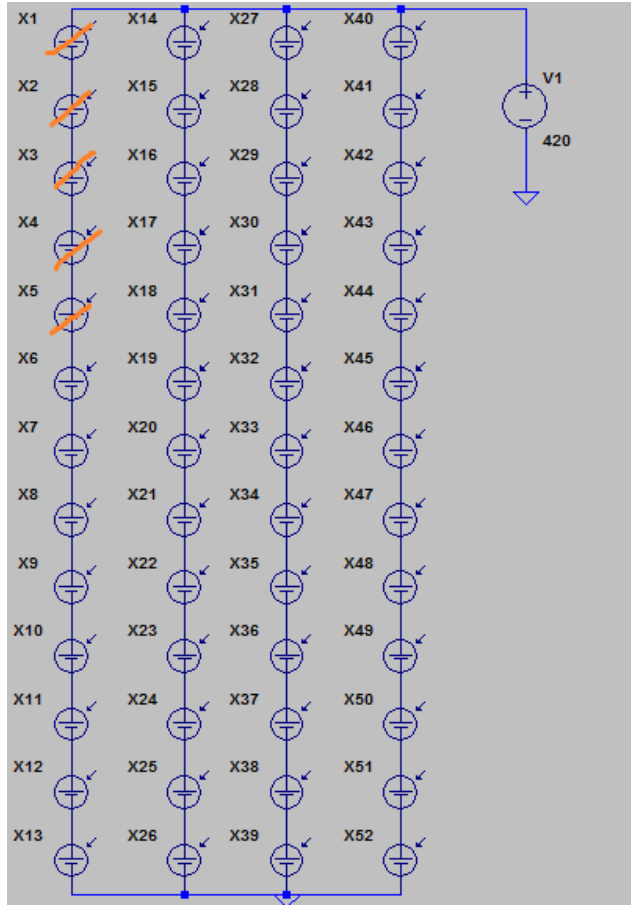

Figure 19: Illustration of shading pattern (a).

that total cross-tied topology performs consistently better than the other topologies. Therefore, TCT is the optimal topology for this shading pattern.

Shading pattern (b), shown in Fig. 20, consists of four shaded modules in the first string and one shaded module in the second string. From the results in Table 5, it is observed that total cross-tied topology performs consistently better than the other topologies. Therefore, TCT is the optimal topology for this shading pattern.

In shading pattern (c), shown in Fig. 21, the shaded modules are spread across all the strings. From the tabulated results in Table 6, it is observed that series-parallel topology performs better for all the shade factors except for $60-90 \%$. For these cases TCT topology performs slightly

\begin{tabular}{|c||c||c||c|}
\hline \multicolumn{1}{|c||}{ Shade factor (\%) } & \multicolumn{3}{c|}{ Percentage output power } \\
\hline \multicolumn{1}{|c||}{10} & SP & TCT & BL \\
20 & 77.7 & 83.5 & 82.6 \\
30 & 80.4 & 85.8 & 85 \\
40 & 83.1 & 88.1 & 87.3 \\
50 & 85.8 & 90.2 & 89.5 \\
60 & 88.4 & 92.2 & 91.7 \\
70 & 91.1 & 94.2 & 93.9 \\
80 & 93.7 & 96.1 & 95.8 \\
90 & 96.3 & 97.6 & 97.5 \\
100 & 98.5 & 98.9 & 98.9 \\
& 100 & 100 & 100 \\
\hline
\end{tabular}

Table 4: Performance of the topologies for the shading pattern (a). 


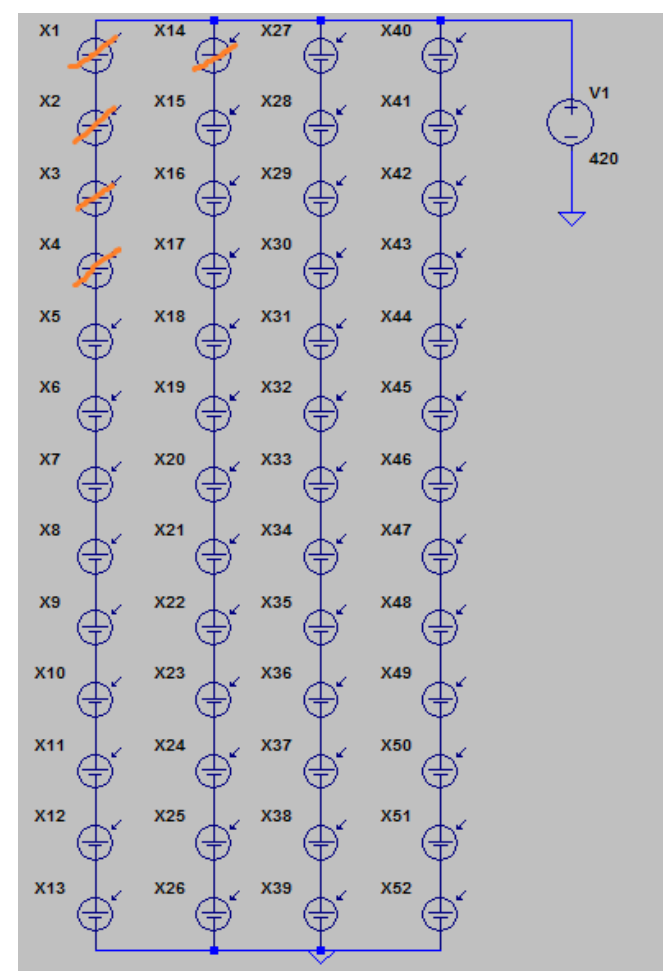

Figure 20: Illustration of shading pattern (b).

better than the SP topology. However, the percentage gain that would be obtained by reconfiguring the topology from SP to TCT for these cases is small compared to the other cases. Therefore, the SP topology may reasonably be considered optimal for this shading pattern.

In shading pattern (d), shown in Fig. 22, the shaded modules are spread across three strings of the array. From the tabulated results in Table 7 , it is observed that seriesparallel topology performs better for all the shade factors except for $70-90 \%$. However, the percentage gain that would be obtained by reconfiguring the topology from SP to TCT for these cases is small compared to the other

\begin{tabular}{|c||c||c||c|}
\hline \multicolumn{1}{|c||}{ Shade factor (\%) } & \multicolumn{3}{c|}{ Percentage output power } \\
\hline & SP & TCT & BL \\
\hline 10 & 75.3 & 77.6 & 77.2 \\
20 & 77.3 & 79.6 & 79.4 \\
30 & 79.9 & 81.7 & 81.5 \\
40 & 82.5 & 83.8 & 83.7 \\
50 & 85.1 & 85.7 & 85.7 \\
60 & 87.7 & 89.4 & 88.2 \\
70 & 90.3 & 93.2 & 92.4 \\
80 & 93.4 & 96.4 & 95.9 \\
90 & 97.9 & 98.7 & 98.7 \\
100 & 100 & 100 & 100 \\
\hline
\end{tabular}

Table 5: Performance of the topologies for the shading pattern (b).

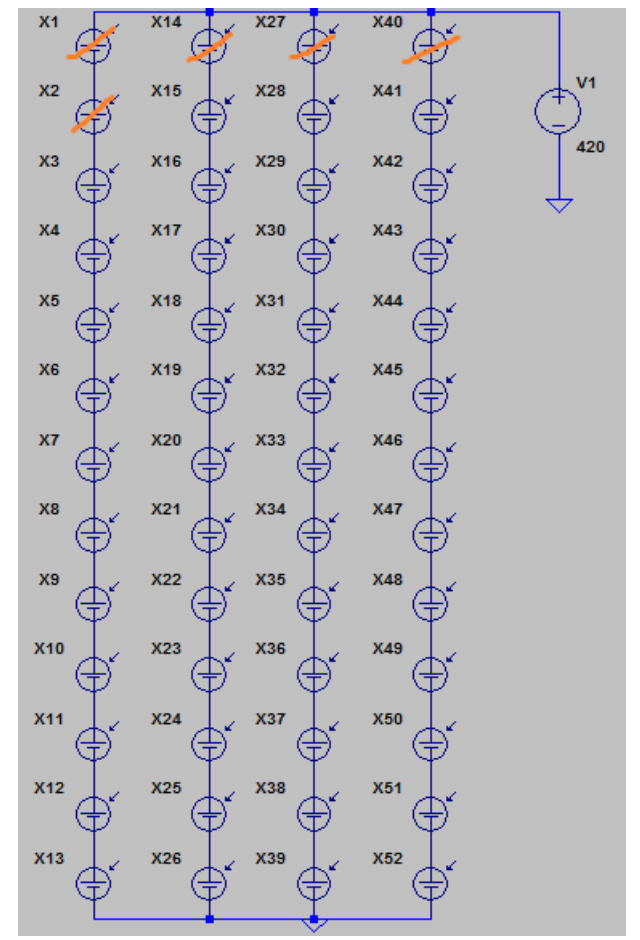

Figure 21: Illustration of shading pattern (c).

\begin{tabular}{|c||c||c||c|}
\hline \multicolumn{1}{|c||}{ Shade factor (\%) } & \multicolumn{3}{c|}{ Percentage output power } \\
\hline \multicolumn{1}{|c||}{} & SP & TCT & BL \\
\hline 10 & 88.9 & 84.4 & 85.7 \\
20 & 88.9 & 84.4 & 85.7 \\
30 & 88.9 & 84.9 & 85.7 \\
40 & 88.9 & 86.7 & 85.7 \\
50 & 89 & 88.1 & 86.6 \\
60 & 89 & 89.7 & 88.3 \\
70 & 89 & 90.7 & 89.7 \\
80 & 89.7 & 91.6 & 91.1 \\
90 & 97.1 & 97.2 & 97.1 \\
100 & 100 & 100 & 100 \\
\hline
\end{tabular}

Table 6: Performance of the topologies for the shading pattern (c). 


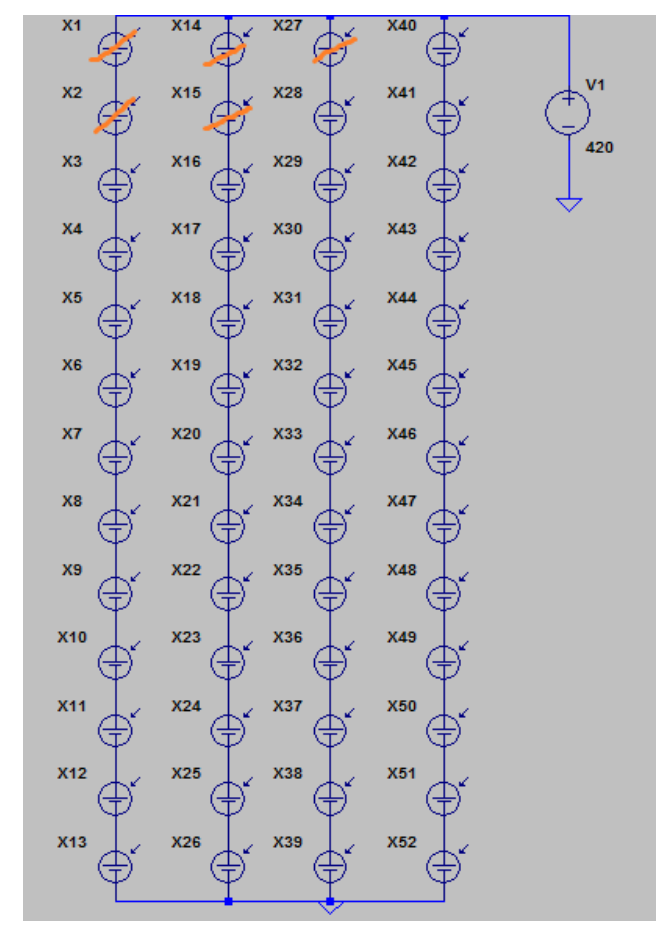

Figure 22: Illustration of shading pattern (d).

cases. Therefore, the SP topology may reasonably be considered optimal for this shading pattern.

In shading pattern (e), shown in Fig. 23, the shaded modules are distributed across half of the total number of strings in the array. From the tabulated results in Table 8 , it is observed that the series-parallel and total crosstied topologies perform significantly better than the other topologies for lower (10 to 40) and higher (50 to 90) shade factors respectively. Therefore for this shading pattern, either SP or TCT is considered as the optimal topology depending on the intensity of shading.

\begin{tabular}{|c||c||c||c|}
\hline Shade factor (\%) & \multicolumn{3}{c|}{ Percentage output power } \\
\hline & SP & TCT & BL \\
\hline 10 & 87.3 & 84.4 & 84.6 \\
20 & 87.3 & 84.4 & 84.6 \\
30 & 87.3 & 84.4 & 84.6 \\
40 & 87.3 & 84.4 & 84.6 \\
50 & 87.3 & 84.4 & 84.6 \\
60 & 87.3 & 84.4 & 84.6 \\
70 & 87.3 & 87.5 & 87.1 \\
80 & 91.2 & 93.5 & 93.3 \\
90 & 97.4 & 98.3 & 98.2 \\
100 & 100 & 100 & 100 \\
\hline
\end{tabular}

Table 7: Performance of the topologies for the shading pattern (d).

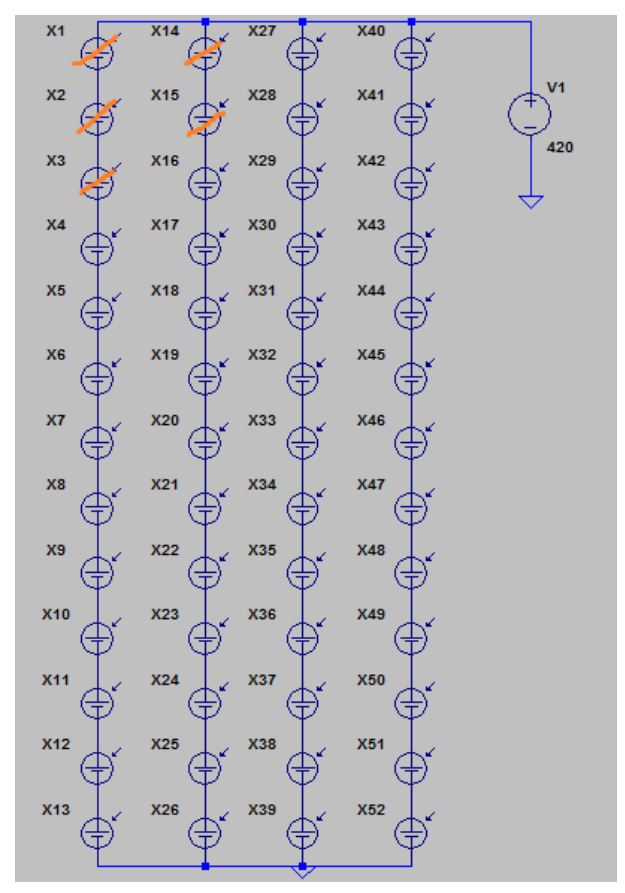

Figure 23: Illustration of shading pattern (e).

\subsection{Analysis}

From the simulation results obtained, it is observed that depending on the shading pattern, either SP or TCT is the optimal topology. Switching between the SP and TCT topologies results in a power increase of $4-6 \%$. This justifies the technique of topology reconfiguration in $\mathrm{PV}$ arrays.

An analysis of the topology performance for the different shading patterns shows that if the shaded modules are:

- localized to a single string, then TCT is the optimal topology.

- spread across all the strings, then SP is the optimal topology.

\begin{tabular}{|c||c||c|c|}
\hline \multicolumn{1}{|c||}{ Shade factor (\%) } & \multicolumn{3}{c|}{ Percentage output power } \\
\hline & SP & TCT & BL \\
\hline 10 & 82.5 & 76.5 & 77.2 \\
20 & 82.5 & 76.5 & 77.2 \\
30 & 82.5 & 77.5 & 77.2 \\
40 & 82.5 & 79.5 & 78.9 \\
50 & 82.5 & 84 & 83.6 \\
60 & 82.7 & 88.7 & 88.2 \\
70 & 88.1 & 92.7 & 92.3 \\
80 & 93.3 & 96.1 & 95.8 \\
90 & 97.9 & 98.7 & 98.6 \\
100 & 100 & 100 & 100 \\
\hline
\end{tabular}

Table 8: Performance of the topologies for the shading pattern (e). 


\begin{tabular}{|c||c||c||c|}
\hline \multicolumn{1}{|c||}{ Shade factor (\%) } & \multicolumn{3}{c|}{ Percentage output power } \\
\hline \multicolumn{1}{|c||}{} & SP & TCT & BL \\
\hline 10 & 82.3 & 82.47 & 82.5 \\
20 & 83.47 & 83.5 & 83.6 \\
30 & 84.8 & 84.7 & 84.7 \\
40 & 86.12 & 86.2 & 85.8 \\
50 & 87.4 & 87.6 & 87.1 \\
60 & 88.7 & 89.4 & 88.7 \\
70 & 90.07 & 91.8 & 91.25 \\
80 & 92.65 & 94.7 & 94.45 \\
90 & 97.72 & 98.2 & 98.22 \\
100 & 100 & 100 & 100 \\
\hline
\end{tabular}

Table 9: Average performance of the topologies assuming equally likely shading patterns.

- distributed across half of the total number of strings in the array, then either SP/ TCT is the optimal topology depending on the intensity of shading.

\subsection{Average Performance}

In this section, an average measure of the topologies' performance is discussed to determine whether any of the topologies would perform consistently better than the others. In reality, the probability of occurrence of shading patterns depends on the physical location, surroundings, and even cloud patterns. If a single topology outperforms the others consistently, then that topology can be fixed and no switching capability is necessary for the array.

The shading patterns shown in Figs. 19 to 23 represent shade scenarios that commonly occur on a PV array in practice. They include the shade patterns caused by chimneys, over head trackers, transmission lines and random cloud patterns. Assume that the shading patterns are equally likely. Under this assumption, the average array power of the topologies for the shading patterns is tabulated in Table 9.

As mentioned in the beginning of this section, the probabilities of the shade patterns is highly dependent on the physical location of the array and the cloud patterns. For a given array at a location, the array performance of the topologies can be studied and the probability of shade patterns can be found. If a particular shade pattern predominantly occurs, then the array can be built with the optimal topology for that pattern and the benefits of switching are negligible. On the other hand, if the shading patterns appear with equal probability, then the topology reconfiguration method can be used to find the performance of topologies and the array configuration can be switched to the optimal topology to get the maximum efficiency from the array.

\subsection{Generalization of the Results}

This section performs a detailed analysis of the array behavior under different kinds of shading patterns. The shading patterns can be broadly classified into three categories, depending on the pattern of shaded modules. The shaded modules can be localized to a single string (Figs. 19 and 20), present across all the strings (refer Figures 21 and 22) and distributed across half of the total number of strings (Fig. 23). The array behavior and optimal topology for each of the shading patterns is analyzed below:

- If shaded modules are present in a single string (e.g. Figs. 19 and 20), then the TCT topology performs better than SP topology. This is because in the SP configuration, the bypass diodes are activated at larger shade factors, corresponding to less severe shading. On the other hand, in the TCT configuration, adjacent healthy modules share the current a shaded module cannot conduct, and the shaded module's bypass diode does not activate until shading is much more severe. These alternate current paths allow the shaded module to continue producing power, reducing overall loss of array output.

- If shaded modules are present across all the strings (e.g. Figs. 21 and 22), then the SP topology outperforms the TCT topology. This is because in the TCT topology, shaded modules are present across an entire row of modules, so no healthy modules are available to compensate for the reduced current through shaded modules. This causes bypass diodes to activate at large irradiance (i.e. mild shading). However, in the SP configuration, string voltages are constrained to be equal. The bypassing of a single module only causes a small change in string voltage, and voltage mismatch losses are much smaller than current mismatch losses in the SP configuration.

- In the third kind of shading pattern, significant number of shaded modules are present in half of the total number of strings (e.g. Fig. 23). This is a variation of the two shading patterns discussed above. In this case, either SP/ TCT is the optimal topology depending on the intensity of shading. The voltage loss from bypassed shaded modules in SP and current loss due to shaded modules in the strings of TCT determines the best topology.

\section{Conclusions}

The topology reconfiguration method presented in this research is used to maximize array power under partial shade conditions. The electrical behavior of the seriesparallel (SP), total cross-tied (TCT), and bridge link (BL) configurations, as well as their bypassed versions, is studied for various shading situations. For all the cases, SPICE circuit simulation is used to find the best configuration and the percentage gain that would be obtained by switching to that configuration. Results show that the optimal topology is determined by which specific modules are shaded. 
Switching to the optimal topology results in a power output increase of up to $4-6 \%$ relative to a non-reconfigurable array under the same conditions. This supports our goal of applying signal processing techniques to PV array management as part of a comprehensive monitoring and optimization strategy $[2,3,4,5,28]$.

The effect of irradiance on the array behavior is analyzed. It is observed that the percentage loss in array power for a given number of shaded modules does not vary significantly with respect to irradiance. For a given number of shaded modules, the maximum percentage deviation in the topologies considered is $1.5 \%$.

The intensity of shading on the array performance is studied by varying the shade factor in the range 10 to 100 $\%$. From the results, it is observed that TCT is the optimal topology when the shaded modules are present in a single string. If the shaded modules are spread across all the strings, then SP is the optimal topology. For the shading patterns in which the shaded modules are distributed across half of the total number of strings in the array, either SP or TCT may be the optimal configuration depending on the intensity of shading.

The method described here has both advantages and disadvantages relative to the irradiance equalization or adaptive banking methods described in Section 3. In both prior methods, the array was restricted to the TCT topology, rendering it more vulnerable to shading occurring across many strings. Adaptive banking is likely to show degraded performance as soon as the number of shaded modules exceeds the size of the adaptive bank, rendering it less useful for widespread but mild shading conditions. Irradiance equalization, on the other hand, assumes an array where any module can be electrically joined to any other module. Implementing this method at scale is an open question as far as the authors are aware. The method described here may be viewed as an attempt to strike a balance between complexity of switching behavior and shading mitigation effectiveness. Like the irradiance equalization method, no upper limit on the number of shaded modules is set. However, like the Adaptive banking method, modules are constrained in terms of who their neighbors are.

A clear next step in this work is to develop partial shading prediction techniques. This would allow an algorithm to fully account for inverter downtime, moving beyond an instantaneous prediction of output power. Work is underway to develop a reliable imaging-based shading prediction system. Another potential improvement would be to optimize AC power output rather than DC maximum power. However, modern inverters are highly efficient across a wide variety of operating conditions [29], and any improvement from optimizing AC is likely to be negligible.

This work contributes a more thorough understanding of the effect of topology on PV arrays under shading conditions and gives a procedure for optimizing the output of an array by dynamically switching the array configuration.
This procedure has the potential to increase array output and therefore lower the cost of PV power.

\section{Bibliography}

[1] J. Conti, P. Holtberg, Levelized cost of new generation resources in the annual energy outlook 2011, Tech. rep., US Energy Information Administration (Dec. 2010)

[2] H. Braun, S. T. Buddha, V. Krishnan, C. Tepedelenlioglu, A. Spanias, T. Yeider, T. Takehara, Signal processing for fault detection in photovoltaic arrays, in: IEEE Int. Conf. on Acoustics, Speech, and Signal Processing, Kyoto, Japan, 2012, pp. $1681-1684$

[3] H. Braun, S. T. Buddha, V. Krishnan, A. Spanias, C. Tepedelenlioglu, T. Takehara, T. Yeider, M. Banavar, Signal Processing for Solar Array Monitoring, Fault Detection, and Optimization, Morgan \& Claypool, 2012, ISBN:1608459489.

[4] S. T. Buddha, H. Braun, V. Krishnan, C. Tepedelenlioglu, A. Spanias, T. Yeider, T. Takehara, Signal processing for photovoltaic arrays, in: Int. Conf. on Emerging Signal Processing Applications, Las Vegas, Nevada, 2012, pp. 115 - 118.

[5] S. Peshin, D. Ramirez, J. Lee, H. Braun., C. Tepedelenlioglu, A. Spanias., M. Banavar, D. Srinivasan, A photovoltaic (PV) array monitoring simulator, in: Modeling, Identification, and control, int. conf. on, Innsbruck, Austria, 2015.

[6] H. Braun, S. Peshin, A. Spanias, C. Tepedelenlioglu, M. Banavar, G. Kalyanasundaram, D. Srinivansan, Irradiance estimation for a smart PV array, in: Energy Conversion Conference and Expo, Montreal, Canada, 2015.

[7] J. Nelson, The Physics of Solar Cells, Imperial College Press, 2003.

[8] W. De Soto, S. Klein, W. Beckman, Improvement and validation of a model for photovoltaic array performance, Solar Energy 80 (1) (2006) 78-88. doi:DOI: 10.1016/j.solener.2005.06.010.

[9] D. King, J. Kratochvil, W. Boyson, Photovoltaic array performance model, Tech. rep., Sandia National Laboratory (2004).

[10] W. D. De Soto, Improvement and validation of a model for photovoltaic array performance, Master's thesis, University of Wisconsin-Madison (2004).

[11] W. D. Soto, EES solver. URL http://sel.me.wisc.edu/software.shtml.

[12] E. Noll, Wind/solar energy for radiocommunications and lowpower electrical systems, H.W. Sams, 1981.

[13] D. Picault, B. Raison, S. Bacha, J. Aguilera, J. De La Casa, Changing photovoltaic array interconnections to reduce mismatch losses: a case study, International Conference on Environment and Electrical Engineering.

[14] G. S. E. Society, Planning and Installing Photovoltaic Systems : A Guide for Installers, Architects and Engineers, 2nd Edition, Earthscan Ltd, 2007.

[15] NFPA 70: National electric code (2011).

[16] G. Velasco, J. Negroni, F. Guinjoan, R. Pique, Irradiance equalization method for output power optimization in plant oriented grid-connected PV generators, In Proc. 2005 European Conference on Power Electronics and Applications (2005) 10.

[17] G. Velasco-Quesada, F. Guinjoan-Gispert, R. Pique-Lopez, M. Roman-Lumbreras, A. Conesa-Roca, Electrical PV array reconfiguration strategy for energy extraction improvement in grid-connected PV systems, IEEE Transactions on Industrial Electronics, 56 (11) (2009) $4319 \quad-4331$. doi:10.1109/TIE.2009.2024664.

[18] G. Velasco, F. Guinjoan, R. Pique, Grid-connected PV systems energy extraction improvement by means of an electric array reconfiguration (EAR) strategy: Operating principle and experimental results, In Proc. IEEE Power Electronics Specialists Conference, 2008. PESC 2008. (2008) 1983 -1988.

[19] D. Nguyen, Modeling and reconfiguration of solar photovoltaic arrays under non-uniform shadow conditions, Master's thesis, Northeastern University (2008). 
[20] D. Nguyen, B. Lehman, A reconfigurable solar photovoltaic array under shadow conditions, In Proc. Twenty-Third Annual IEEE Applied Power Electronics Conference and Exposition, 2008. APEC 2008. (2008) $980-986$.

[21] R. Candela, V. Di Dio, E. Riva Sanseverino, P. Romano, Reconfiguration techniques of partial shaded pv systems for the maximization of electrical energy production, In Proc. International Conference on Clean Electrical Power, 2007. ICCEP '07. (2007) $716-719$.

[22] D. Picault, B. Raison, S. Bacha, J. de la Casa, J. Aguilera, Forecasting photovoltaic array power production subject to mismatch losses, Solar Energy 84 (7) (2010) 1301 - 1309. doi:10.1016/j.solener.2010.04.009.

[23] N. D. Kaushika, N. K. Gautam, Energy yield simulations of interconnected solar PV arrays, IEEE Power Engineering Review, 22 (8) (2002) 62 -62. doi:10.1109/MPER.2002.4312475.

[24] N. Kaushika, A. K. Rai, An investigation of mismatch losses in solar photovoltaic cell networks, Energy 32 (5) (2007) 755 759. doi:10.1016/j.energy.2006.06.017.

[25] IEEE 1547: Standard for interconnecting distributed resources with electric power systems (2003).

[26] Hitachi SJ200 Series Inverter Instruction Manual.

[27] Fuji Electric, FVR-Micro Instruction Manual.

[28] A. Spanias, Signal Processing: An Interactive Approach, 2nd Edition, LuLu.com, 2014, ISBN 978-1-4675-9892-7.

[29] D. L. King, S. Gonzalez, G. M. Galbraith, W. E. Boyson, Performance model for grid-connected photovoltaic inverters, Tech. rep., Sandia National Laboratory (2007). 